\title{
DETECTING COALESCENCES OF INTERMEDIATE-MASS BLACK HOLES IN GLOBULAR CLUSTERS WITH THE EINSTEIN TELESCOPE
}

\author{
I. MANDEL \\ NSF Astronomy and Astrophysics Postdoctoral Fellow \\ Department of Physics and Astronomy, Northwestern University \\ Evanston, IL 60208 \\ ilyamandel@chgk.info \\ J. R. GAIR \\ Institute of Astronomy, University of Cambridge \\ Cambridge, CB3OHA, UK \\ M. C. MILLER \\ Department of Astronomy and Center for Theory and Computation, University of Maryland \\ College Park, MD 20742
}

\begin{abstract}
We discuss the capability of a third-generation ground-based detector such as the Einstein Telescope (ET) to detect mergers of intermediate-mass black holes (IMBHs) that may have formed through runaway stellar collisions in globular clusters. We find that detection rates of $\sim 500$ events per year are plausible 1

Keywords: Gravitational Waves; Intermediate-Mass Black Holes; the Einstein Telescope.
\end{abstract}

The Einstein Telescope (ET), a proposed third-generation ground-based gravitational-wave $(\mathrm{GW})$ detector, will be able to probe GWs in a frequency range

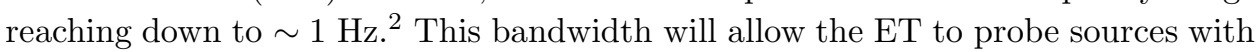
masses of hundreds or a few thousand $M_{\odot}$ which are out of reach of LISA or the current ground-based detectors LIGO, Virgo, and GEO-600.

Globular clusters may host intermediate-mass black holes (IMBHs) with masses in the $\sim 100-1000 M_{\odot}$ range (see Ref. 3 and references therein). If the stellar binary fraction in a globular cluster is sufficiently high, two or more IMBHs can form. 4 These IMBHs then sink to the center in a few million years, where they form a binary and merge via three-body interactions with cluster stars followed by gravitational radiation reaction ( $\mathrm{sec}^{\sqrt{45}}$ for more details). Therefore, the rate of IMBH binary mergers is just the rate at which pairs of IMBHs form in clusters. The rate of detectable coalescences is

$$
R \equiv \frac{d N_{\mathrm{event}}}{d t_{o}}=\int_{M_{\mathrm{tot}, \min }}^{M_{\mathrm{tot}, \max }} d M_{\mathrm{tot}} \int_{0}^{1} d q \int_{0}^{z_{\max }\left(M_{\mathrm{tot}}, q\right)} d z \frac{d^{4} N_{\mathrm{event}}}{d M_{\mathrm{tot}} d q d t_{e} d V_{c}} \frac{d t_{e}}{d t_{o}} \frac{d V_{c}}{d z} .
$$

Here $M_{\text {tot }}$ is the total mass of the coalescing IMBH-IMBH binary and $q \leq 1$ is the mass ratio between the IMBHs; $z_{\max }\left(M_{\text {tot }}, q\right)$ is the maximum redshift to which the ET could detect a merger between two IMBHs of total mass $M_{\text {tot }}$ and mass ratio $q$; $d t_{e} / d t_{o}=(1+z)^{-1}$ is the relation between local time and our observed time, and 
$d V_{c} / d z$ is the change of comoving volume with redshift, given by

$$
\frac{d V_{c}}{d z}=4 \pi D_{H}^{3}\left[\Omega_{M}(1+z)^{3}+\Omega_{\Lambda}\right]^{-1 / 2}\left\{\int_{0}^{z} \frac{d z^{\prime}}{\left[\Omega_{M}\left(1+z^{\prime}\right)^{3}+\Omega_{\Lambda}\right]^{1 / 2}}\right\}^{2} .
$$

We assume a flat universe $\left(\Omega_{k}=0\right)$, and use $\Omega_{M}=0.27, \Omega_{\Lambda}=0.73, H_{0}=$ $72 \mathrm{~km} \mathrm{~s}^{-1} \mathrm{Mpc}^{-1}$, and $D_{H}=c / H_{0} \approx 4170 \mathrm{Mpc}$, so that the luminosity distance can be written as a function of redshift as $![$

$$
D_{L}(z)=D_{H}(1+z)\left\{\int_{0}^{z} \frac{d z^{\prime}}{\left[\Omega_{M}\left(1+z^{\prime}\right)^{3}+\Omega_{\Lambda}\right]^{1 / 2}}\right\} .
$$

We make the following assumptions. 1. IMBH pairs form in a fraction $g$ of all globular clusters. 2. We neglect the delay between cluster formation and IMBH coalescence. 3. When an IMBH pair forms in a cluster, its total mass is a fixed fraction of the cluster mass, $M_{\mathrm{tot}}=2 \times 10^{-3} M_{\mathrm{cl}}$, consistent with simulations ${ }^{7}$ The mass ratio is uniform in $[0,1]$. We restrict our attention to systems with a total mass between $M_{\text {tot,min }}=100 M_{\odot}$ and $M_{\text {tot,max }}=20000 M_{\odot}$. Thus,

$$
\frac{d^{4} N_{\text {event }}}{d M_{\text {tot }} d q d t_{e} d V_{c}}=g \frac{d^{3} N_{\mathrm{cl}}}{d M_{\mathrm{cl}} d t_{e} d V_{c}} \frac{1}{2 \times 10^{-3}} .
$$

4. The distribution of cluster masses scales as $\left(d N_{\mathrm{cl}} / d M_{\mathrm{cl}}\right) \propto M_{\mathrm{cl}}^{-2}$ independently of redshift. We confine our attention to clusters with masses ranging from $M_{\mathrm{cl} \text {,min }}=$ $5 \times 10^{4} M_{\odot}$ to $M_{\mathrm{cl}, \max }=10^{7} M_{\odot}$. The total mass formed in all clusters in this mass range at a given redshift is a redshift-independent fraction $g_{\mathrm{cl}}$ of the total star formation rate per comoving volume:

$$
\frac{d^{3} N_{\mathrm{cl}}}{d M_{\mathrm{cl}} d t_{e} d V_{c}}=\frac{g_{\mathrm{cl}}}{\ln \left(M_{\mathrm{cl}, \max } / M_{\mathrm{cl}, \min }\right)} \frac{d^{2} M_{\mathrm{SF}}}{d V_{c} d t_{e}} \frac{1}{M_{\mathrm{cl}}^{2}}
$$

5. The star formation rate as a function of redshift $z$ rises rapidly with increasing $z$ to $z \sim 2$, after which it remains roughly constant $\underline{8}$

$$
\frac{d^{2} M_{\mathrm{SF}}}{d V_{c} d t_{e}}=0.17 \frac{e^{3.4 z}}{e^{3.4 z}+22} \frac{\left[\Omega_{M}(1+z)^{3}+\Omega_{\Lambda}\right]^{1 / 2}}{(1+z)^{3 / 2}} M_{\odot} \mathrm{yr}^{-1} \mathrm{Mpc}^{-3} .
$$

Rather than computing $z_{\max }\left(M_{\mathrm{tot}}, q\right)$ [Eq. 1] for all values of $M_{\text {tot }}$ and $q$, we rely on the following fitting formula for the luminosity-distance range $D_{\mathrm{L}, \max }$ as a function of the redshifted total mass $M_{z}=M_{\text {tot }}(1+z)$, obtained by using the effectiveone-body, numerical relativity (EOBNR) gravitational waveforms ${ }^{9}$ to model the inspiral, merger, and ringdown phases of coalescence:

$$
D\left(M_{z}\right)=(A \mathrm{Mpc})\left\{\begin{array}{ll}
\left(M_{z} / M_{\odot}\right)^{3 / 5} & \text { if } M_{z}<M_{0} \\
\left(M_{0} / M_{\odot}\right)^{11 / 10}\left(M_{z} / M_{\odot}\right)^{-1 / 2} & \text { if } M_{z}>M_{0}
\end{array},\right.
$$

where $A=500, M_{0}=600 M_{\odot}$ for $q=1$ and $A=281, M_{0}=450 M_{\odot}$ for $q=0.25$. We use $\rho=8$ as the SNR threshold for a "single ET" configuration. We determine the sky-location and orientation averaged range by dividing the horizon distance by $2.26 \stackrel{10}{10}$ ignoring redshift corrections to this factor. 
We can compute $z\left(D_{L}\right)$ by inverting Eq. (3). For a given choice of $M_{\text {tot }}$ and $q$, the maximum detectable redshift $z_{\max }\left(M_{\text {tot }}, q\right)$ is then obtained by finding a self-consistent solution of $z\left(D_{\mathrm{L}, \max }\left(M_{\mathrm{tot}}\left(1+z_{\max }\right)\right)\right)=z_{\max }$.

In order to compute the rate of detectable coalescences, we carry out the integrals over $M_{\text {tot }}$ and $z$ in Eq. (11) for two specific values of $q$. For $q=1$, we find the total

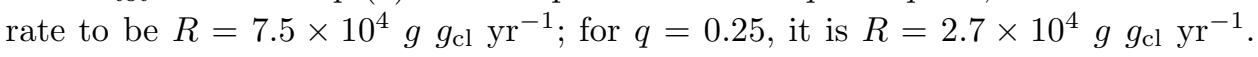
The range varies smoothly with $q$; therefore, we estimate that full rate, including the integral over $q$ is

$$
\begin{aligned}
R= & \frac{2 \times 10^{-3} g g_{\mathrm{cl} \mathrm{yr}^{-1}}}{\ln \left(M_{\mathrm{tot}, \max } / M_{\mathrm{tot}, \min }\right)} \int_{M_{\mathrm{tot}, \min }}^{M_{\mathrm{tot}, \max }} \frac{M_{\odot} d M_{\mathrm{tot}}}{M_{\mathrm{tot}}^{2}} \int_{0}^{1} d q \\
& \int_{0}^{z_{\max }\left(M_{\mathrm{tot}}, q\right)} d z 0.17 \frac{e^{3.4 z}}{e^{3.4 z}+22} \frac{4 \pi\left(D_{H} / \mathrm{Mpc}\right)^{3}}{(1+z)^{5 / 2}} \times\left\{\int_{0}^{z} \frac{d z^{\prime}}{\left[\Omega_{M}\left(1+z^{\prime}\right)^{3}+\Omega_{\Lambda}\right]^{1 / 2}}\right\}^{2} \\
\approx & 500\left(\frac{g}{0.1}\right)\left(\frac{g_{\mathrm{cl}}}{0.1}\right) \mathrm{yr}^{-1},
\end{aligned}
$$

where we arbitrarily chose $g=0.1$ and $g_{\mathrm{cl}}=0.1$ as the default scalings.

Mergers between pairs of globular clusters containing IMBHs can increase this rate by up to a factor of $\sim 2 !^{[1]}$ Ref. 1 contains additional details on coalescences involving intermediate-mass black holes as gravitational-wave sources for the ET.

\section{Acknowledgments}

IM is supported by the NSF Astronomy and Astrophysics Postdoctoral Fellowship under award AST-0901985 and was partially supported from NASA ATP Grant NNX07AH22G. JG's work is supported by a Royal Society University Research Fellowship. MCM acknowledges NASA ATP grant NNX08AH29G. IM's participation in MG12 was enabled by an NSF travel grant.

\section{References}

1. J. R. Gair, I. Mandel, M. C. Miller and M. Volonteri, ArXiv e-prints (2009), 0907.5450.

2. A. Freise, S. Chelkowski, S. Hild, W. Del Pozzo, A. Perreca and A. Vecchio, Classical and Quantum Gravity 26, 085012 (2009).

3. M. C. Miller, ArXiv e-prints (2008), 0812.3028.

4. J. M. Fregeau, S. L. Larson, M. C. Miller, R. O'Shaughnessy and F. A. Rasio, Astrophysical Journal Letters 646, L135 (2006).

5. P. Amaro-Seoane, J. R. Gair, M. Freitag, M. C. Miller, I. Mandel, C. J. Cutler and S. Babak, Classical and Quantum Gravity 24, 113 (2007).

6. D. W. Hogg, ArXiv Astrophysics e-prints (1999), astro-ph/9905116.

7. M. A. Gürkan, M. Freitag and F. A. Rasio, Astrophysical Journal 604, 632 (2004).

8. C. C. Steidel, K. L. Adelberger, M. Giavalisco, M. Dickinson and M. Pettini, Astrophysical Journal 519, 1 (1999).

9. A. Buonanno, Y. Pan, J. G. Baker, J. Centrella, B. J. Kelly, S. T. McWilliams and J. R. van Meter, Phys. Rev. D 76, 104049 (2007).

10. L. S. Finn and D. F. Chernoff, Phys. Rev. D 47, 2198 (1993).

11. P. Amaro-Seoane and L. Santamaria, ArXiv e-prints (2009), 0910.0254. 